\title{
Attachment of Borrelia burgdorferi within Ixodes scapularis mediated by outer surface protein $A$
}

\author{
Utpal Pal, ${ }^{1}$ Aravinda M. de Silva, ${ }^{2}$ Ruth R. Montgomery, ${ }^{1}$ Durland Fish, ${ }^{3}$ \\ Juan Anguita, ${ }^{1}$ John F. Anderson, ${ }^{4}$ Yves Lobet,${ }^{5}$ and Erol Fikrig' ${ }^{1}$
}

\begin{abstract}
${ }^{1}$ Section of Rheumatology, Department of Internal Medicine, Yale University School of Medicine, New Haven, Connecticut, USA ${ }^{2}$ Department of Microbiology and Immunology, University of North Carolina at Chapel Hill, Chapel Hill, North Carolina, USA ${ }^{3}$ Department of Epidemiology and Public Health, Yale University School of Medicine, New Haven, Connecticut, USA ${ }^{4}$ Department of Entomology, Connecticut Agricultural Experiment Station, New Haven, Connecticut, USA ${ }^{5}$ SmithKline Beecham Biologicals, Rixensart, Belgium
\end{abstract}

Address correspondence to: Erol Fikrig, 608 Laboratory of Clinical Investigation, Section of Rheumatology, Department of Internal Medicine, Yale University School of Medicine, 333 Cedar Street, New Haven, Connecticut 06520-8031, USA. Phone: (203) 785-2453; Fax: (203) 785-7053; E-mail: erol.fikrig@yale.edu.

Received for publication January 21, 2000, and accepted in revised form July 6, 2000.

\begin{abstract}
Borrelia burgdorferi outer surface protein (Osp) A has been used as a Lyme disease vaccine that blocks transmission: OspA antibodies of immune hosts enter ticks during blood feeding and destroy spirochetes before transmission to the host can occur. B. burgdorferi produce OspA in the gut of unfed Ixodes scapularis ticks, and many spirochetes repress OspA production during the feeding process. This preferential expression suggests that OspA may have an important function in the vector. Here we show that OspA mediates spirochete attachment to the tick gut by binding to an I. scapularis protein. The binding domains reside in the central region and COOH-terminus of OspA. OspA also binds to itself, suggesting that spirochete-spirochete interactions may further facilitate adherence in the gut. OspA-mediated attachment in the tick provides a possible mechanism for how stage-specific protein expression can contribute to pathogenesis during the B. burgdorferi natural cycle.
\end{abstract}

J. Clin. Invest. 106:561-569 (2000).

\section{Introduction}

Lyme disease is the most common arthropod-borne illness in the United States $(1,2)$. Outer surface protein (Osp) A elicits immunity against Borrelia burgdorferi infection and is being used as a human vaccine (3-6). OspA antibody blocks spirochete transmission to the vertebrate host by binding to $B$. burgdorferi within the gut of engorging Ixodes scapularis ticks (7-10). Phase III clinical trials demonstrate that vaccination with OspA provides $80-90 \%$ protection against Lyme disease, and vaccine failures are generally associated with low antibody responses to OspA in vaccine recipients $(5,6)$.

The temporal expression of ospA during the B. burgdorferi life cycle implies that OspA has a function within the arthropod host. Uninfected larval ticks acquire spirochetes while feeding on infected mice. B. burgdorferi produce OspA soon after leaving the vertebrate host and entering I. scapularis, suggesting an important role for OspA in vector colonization (7-10). Within nymphal ticks, spirochetes abundantly express osp $A$ and remain in close association with the tick gut. When nymphs engorge on a vertebrate host, $B$. burgdorferi in the gut rapidly multiply, and $70-80 \%$ of the organisms clear OspA from their surface (7).

$B$. burgdorferi continues to repress osp $A$ in the mammalian host. ospA mRNA is not readily detectable after tick-borne transmission of spirochetes to mice but can sometimes be found up to 14 days after syringe challenge of mice with OspA-producing B. burgdorferi
(11-15). ospA is also not usually expressed during human infection. However, some individuals may develop humoral and cellular OspA responses in early-stage disease (16-19), and immune responses to OspA have been associated with chronic Lyme arthritis, indicating that this antigen is present in some patients $(20,21)$. The selective expression of osp $A$ when $B$. burgdorferi goes into and resides within I. scapularis and the subsequent general downregulation of osp $A$ when spirochetes exit the tick gut suggest that OspA may play an important role in B. burgdorferi colonization of the arthropod vector.

\section{Methods}

B. burgdorferi and I. scapularis: culture and cultivation

A clonal isolate of B. burgdorferi N40 that is infectious and pathogenic in mice, and represents a prototypic B. burgdorferi sensu stricto organism, was used throughout these studies (22). Spirochetes were cultivated in Barbour-Stoenner-Kelly (BSK) II medium at $33^{\circ} \mathrm{C}(23)$. B. burgdorferi 46047 , a naturally occurring $B$. burgdorferi sensu stricto isolate that lacks OspA (24), was also used in some spirochete-OspA binding studies.

Mated adult female I. scapularis were collected in the field. The egg mass was then laid in the laboratory. Hatched larvae were fed on uninfected $\mathrm{C} 3 \mathrm{H}$ mice to produce pathogen-free nymphs. All tick rearing was performed in an incubator at $26^{\circ} \mathrm{C}$ with $85 \%$ relative humidity and a 12-hour light/dark photo period regimen. 


\section{ELISA}

Protein binding to the tick gut extract. Recombinant OspAs from B. burgdorferi isolates 25015 and N40, and OspC from $B$. burgdorferi $\mathrm{N} 40$, were expressed and purified in their nonlipidated forms $(25,26,27)$. OspA and OspC were expressed either without a fusion partner or as fusion proteins with glutathione transferase (after which the fusion partner was cleaved using thrombin), as described previously $(15,25,26)$. OspA with mutations in specific amino acids, produced using sitedirected mutagenesis (see later in this section), were also expressed as a fusion partner with glutathione transferase, and the fusion partner was subsequently removed with thrombin. Overlapping 18- or 19-mer OspA peptides were synthesized by A. Michel at the University of Mons, Belgium. Peptides were solubilized in DMSO and diluted in PBS. OspAs, OspA peptides, OspC, or BSA was labeled with FITC from Molecular Probes Inc. (Eugene, Oregon, USA). The extent of conjugation of FITC per molecule of protein was determined according to the manufacturer's instructions. One milligram of each FITC-labeled protein represents $34 \mathrm{pmol}$ of OspA, $43 \mathrm{pmol}$ of OspC, and $15 \mathrm{pmol}$ of BSA. One picomole of OspA, OspC, and BSA bound to 2, 2.4, and 6.8 pmol of FITC, respectively.

Guts from flat nymphal I. scapularis ticks were dissected in PBS and homogenized on ice with a Kontes microhomogenizer (VWR Scientific Products, West Chester, Pennsylvania, USA). Salivary glands from nymphal ticks were dissected in PBS and also homogenized on ice. One gut extract equivalent $(0.5 \mu \mathrm{g})$ of protein or $0.5 \mu \mathrm{g}$ of salivary gland protein extract per well was used to coat microtiter plates (ICN Biomedical Inc., Costa Mesa, California, USA). Protein concentrations were determined

\section{Table 1}

The overlapping synthetic OspA peptides

\begin{tabular}{|c|c|c|}
\hline Peptide number & Amino acid sequence & $\begin{array}{l}\text { Amino acid } \\
\text { position }\end{array}$ \\
\hline 1 & CKQNVSSLDEKNSVSVDL & $17-34$ \\
\hline 2 & KNSVSVDLPGEMNVLVSK & $27-44$ \\
\hline 3 & EMNVLVSKEKNKDGKYDL & $37-54$ \\
\hline 4 & KDGKYDLIATVDKLELKG & $48-65$ \\
\hline 5 & KLELKGTSDKNNGSGVLEG & $60-78$ \\
\hline 6 & GSGVLEGVKADKSKVKLTI & $72-90$ \\
\hline 7 & KVKLTISDDLGQTTLEVFK & $85-103$ \\
\hline 8 & LEVFKEDGKTLVSKKVTSK & 99-117 \\
\hline 9 & KVTSKDKSSTEEKFNEKG & $113-130$ \\
\hline 10 & KFNEKGEVSEKIITRADGT & $125-143$ \\
\hline 11 & RADGTRLEYTEIKSDGSG & $139-156$ \\
\hline 12 & KSDGSGKAKEVLKSYVLEG & $151-169$ \\
\hline 13 & YLEGTLTAEKTTLVVKE & $165-182$ \\
\hline 14 & LVVKEGTVTLSKNISKSG & $178-195$ \\
\hline 15 & KNISKSGEVSVELNDTDSSA & $189-208$ \\
\hline 16 & TDSSAATKKTAAWNSGTS & $204-221$ \\
\hline 17 & NSGTSTLTITVNSKKTKD & $217-234$ \\
\hline 18 & SKKTKDLVFTKENTITVQQ & $229-247$ \\
\hline 19 & ITVQQYDSNGTKLEGSAV & $243-260$ \\
\hline 20 & EGSAVEITKLDEIKNALK & $256-273$ \\
\hline
\end{tabular}

Single-letter abbreviations for the amino acid residues: A, Ala; C, Cys; D, Asp; E, Glu; F, Phe; G, Gly; H, His; I, Ile; K, Lys; L, Leu; M, Met; N, Asn; P, Pro; Q, Gln; R, Arg; S, Ser; T, Thr; V, Val; W, Trp; and Y, Tyr. using the Bio-Rad Protein Assay Kit (Bio-Rad Laboratories Inc., Hercules, California, USA). In some studies, the tick gut extract (TGE) in PBS was incubated with a combination of protease inhibitors (Sigma protease inhibitor cocktail, catalog no. P 8340; Sigma Chemical Co., St. Louis, Missouri, USA) to ensure that the potential release of proteases from the intestine would not degrade a putative receptor. The Sigma cocktail contains inhibitors with broad specificity for the inactivation of serine, cysteine, aspartic proteases, and aminopeptidases. One hundred microliters of each extract $(5 \mu \mathrm{g} / \mathrm{mL})$ in PBS was used to coat the wells. As controls, plates were coated (100 $\mu \mathrm{L} /$ well) with $10 \mu \mathrm{g} / \mathrm{mL}$ of BSA, gelatin, or FCS in a similar fashion. Plates were incubated overnight at $4{ }^{\circ} \mathrm{C}$, and tightly covered with cellophane to prevent evaporation. Plates were then washed three time with PBS with $0.05 \%$ Tween 20 (PBS-Tween-20). Nonspecific sites were blocked by incubating the TGE-coated wells with $15 \%$ normal FBS for 2 hours at $37^{\circ} \mathrm{C}$. Plates were then incubated with $100 \mu \mathrm{L}$ of FITC-labeled OspA, OspC, or BSA protein $(10 \mu \mathrm{g} / \mathrm{mL})$ or OspA peptide $(5 \mu \mathrm{g} / \mathrm{mL})$ at $37^{\circ} \mathrm{C}$ for 1 hour. A dose-response curve was also performed using $100 \mu \mathrm{L}$ of FITC-labeled OspA, OspC, and BSA at 5, 10,20 , and $40 \mu \mathrm{g} / \mathrm{mL}$. The plates were washed three times with PBS-Tween-20. Binding was detected using anti-FITC IgG-horseradish peroxidase (Amersham Pharmacia Biotech, Piscataway, New Jersey, USA) as a secondary reagent and TMB microwell peroxidase substrate (KPL, Gaithersburg, Maryland, USA) was used for color development. The OD was read at $450 \mathrm{~nm}$ at either 5 or 15 minutes. In most of the studies, the OD was measured at 15 minutes, at which time the highest recorded values usually ranged between 0.5 and 1.0. In some studies (see Figures $1 \mathrm{~b}$ and $3 \mathrm{a}$ ), the OD values were recorded at 5 minutes because the values obtained with high concentrations of OspA approached the upper limits of detection of the assay.

Spirochete binding to TGE. To examine B. burgdorferi binding to the tick gut, the prepared wells containing TGE were incubated for 4 hours at $30^{\circ} \mathrm{C}$ with $10^{7} \mathrm{~B}$. burgdorferi N40 per well in PBS-Tween 20 supplemented with $5 \%$ FBS. Control wells were coated $(100 \mu \mathrm{L} /$ well $)$ with BSA $(10 \mu \mathrm{g} / \mathrm{mL})$ or FCS $(10 \mu \mathrm{g} / \mathrm{mL})$. Unbound spirochetes were washed away using PBS-Tween. Then a mouse mAb (mAb H9724) directed against FlaB (28) was added to the wells at a dilution of 1:25 and incubated for 1 hour at $30^{\circ} \mathrm{C}$. Anti-mouse IgG-HRP (Sigma Chemical Co.) was used as secondary reagent at a dilution of 1:2,000. TMB microwell peroxidase substrate (KPL) was used for color development, and the OD was read at $450 \mathrm{~nm}$. In some experiments, spirochetes were incubated in the presence of $50 \mu \mathrm{g} /$ well OspA.

Additional studies were performed in an identical fashion, except that PBS was used instead of PBS-Tween, in order to minimize permeabilization of the outer membrane. In these studies, a murine B. burgdorferi antisera was used to detect spirochetes.

OspA-OspA binding. Microtiter wells were coated overnight at $4^{\circ} \mathrm{C}$ with $10 \mu \mathrm{g} /$ well of OspA from $B$. 
burgdorferi N40, OspA from B. burgdorferi 25015, or BSA in PBS. Nonspecific sites were blocked with $15 \%$ FBS, and the plates were probed with $100 \mu \mathrm{L}$ of $10 \mu \mathrm{g} / \mathrm{mL}$ FITC-labeled B. burgdorferi N40 OspA or B. burgdorferi 25015 OspA for 1 hour at $37^{\circ} \mathrm{C}$.

Site-directed mutagenesis

The results suggest that conserved amino acids between B. burgdorferi in the amino acid regions $\mathrm{OspA}_{85-103}$ or $\mathrm{OspA}_{229-247}$ (see Figure 6d) may facilitate binding. Therefore, six OspAs, designated M1, M2, M3, M4, M5, and $\mathrm{M6}$, which carry unique mutations in peptide sequence $\mathrm{OspA}_{85-103}$ or $\mathrm{OspA}_{229-247}$ (Table 1), were created by sitedirected mutagenesis. The amino acid number and sequence in single-letter abbreviation in wild-type $B$. burgdorferi N40 and mutant OspAs are as follows, and encompass all the conserved amino acids in these regions: M1 (KVK85-87 to $\triangle \mathrm{EVE}$ ), M2 (DL93-94 to $\triangle \mathrm{AR}$ ), M3 (EVFK100-103 to $\triangle$ AVFA), M4 (K231 to $\Delta$ A), M5 (VF236-237 to $\Delta \mathrm{GS}$ ), M6 (TIT242-244 to $\Delta$ ANA).

A pGEX-2T-based vector was used to express wildtype or mutant OspAs. Mutant OspAs were generated using the QuickChange site-directed mutagenesis kit (Stratagene, La Jolla, California, USA). The following mutagenic primers were used, and the substitution of codons or nucleotides is indicated by underlining and boldfaced type, respectively. All the mutations were confirmed by sequencing, and wild-type or mutant OspAs were expressed, purified, and labeled with FITC for binding studies as described previously.

M1 (5'cttgaaggegtaaaagctgacaaaagtgaagtagaattaacaatttctgacgatc $3^{\prime}$ and $5^{\prime}$ gatcgtcagaaattgttaattctacttcacttttgtcagcttttacgccttcaag $\left.3^{\prime}\right)$

M2 (5' gtaaagtaaaattaacaatttctgacgctcgaggtcaaaccacacttg3'and $5^{\prime}$ 'aagtgtggtttgacctcgagcgtcagaaattgttaattttactttac3')

M3 (5'ggtcaaaccacacttgcagttttcgcagaagatggcaaaacac $3^{\prime}$ and $5^{\prime}$ gtgttttgccatcttctgcgaaaactgcaagtgtggtttgacc $3^{\prime}$ )

M4 (5'caactttaacaattactgtaaacagtaaagcaactaaagaccttgtgtttacaaaag $3^{\prime}$ and $5^{\prime}$ cttttgtaaacacaaggtctttgttgctttactgtttacagtaattgttaaagttg $\left.3^{\prime}\right)$

M5 (5'ctgtaaacagtaaaaaaactaaagaccttgggtctacaaaagaaaacacaattacag $3^{\prime}$ and $5^{\prime}$ ctgtaattgtgttttcttttgtagacccaaggtctttagtttttttactgtttacag $\left.3^{\prime}\right)$

M6 (5'gaccttgtgtttacaaaagaaaacgcaaatgcagtacaacaatacgac3' and $5^{\prime}$ gtcgtattgttgtactgcatttgcgttttcttttgtaaacacaaggtc $\left.3^{\prime}\right)$.

Confocal microscopy and immunofluorescence

Five to ten midguts were dissected out from nymphal ticks in $100 \mu \mathrm{L}$ of PBS. The organs were cut into two pieces and placed on silylated glass slides (PGC Scientific, Gaithersburg, Maryland, USA) to enhance attachment. Slides were washed twice with PBS, incubated with PBS-Tween 20 with $5 \%$ FBS for 30 minutes at room temperature, and then incubated for 1 hour at room temperature with FITC-labeled OspA, OspC, or BSA (50 $\mu \mathrm{L}$ of $100 \mu \mathrm{g} / \mathrm{mL}$ of FITC-labeled protein). Samples were subsequently stained with propidium iodide (50 $\mu \mathrm{L}$ of a $10 \mu \mathrm{g} / \mathrm{mL}$ solution) for 3 minutes at room temperature, washed three times with PBS-Tween-20, and mounted in glycerol for examination. The tissues were viewed using a Zeiss LSM 510 scanning laser confocal microscope equipped with an argon/krypton laser (Carl Zeiss Inc., Thornwood, New York, USA).

For immunofluorescence, $B$. burgdorferi $\left(10^{7}\right.$ spirochetes $/ \mathrm{mL}$ ) were suspended in PBS, and $10-\mu \mathrm{L}$ aliquots were placed on silylated glass slides and

a
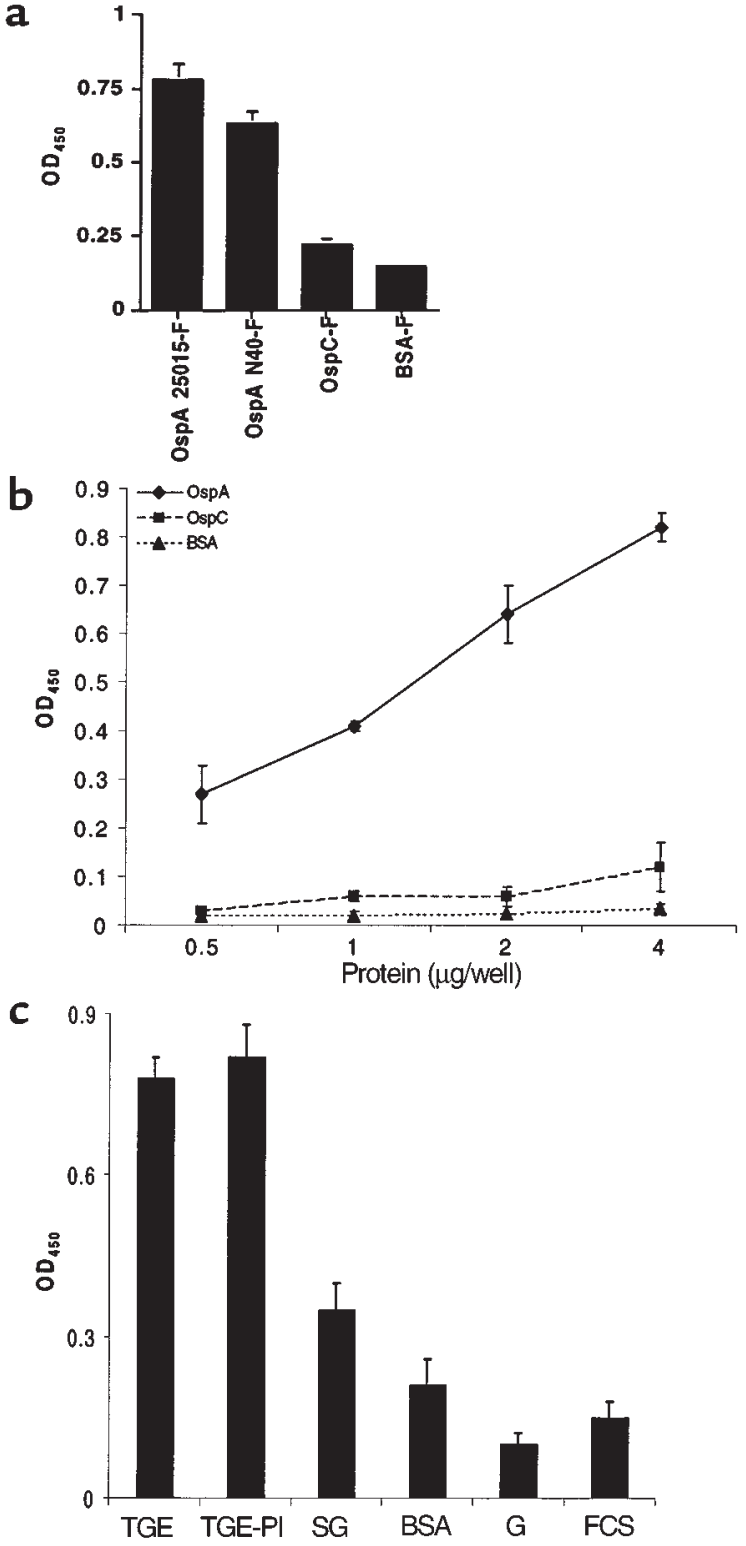

Figure 1

OspA binds to an I. scapularis TGE. (a) FITC-labeled (-F) OspA from B. burgdorferi 25015 or $\mathrm{N} 40$, OspC from B. burgdorferi $\mathrm{N} 40$, and BSA were used to probe TGE-coated wells. Bars represent the $\mathrm{OD}_{450}$ at 15 minutes (mean $\pm \mathrm{SD}$ ) from three experiments. (b) Dose-response curves are presented for FITC-labeled OspA-N40, OspC, and BSA binding to TGE. The $\mathrm{OD}_{450}$ was measured at 5 minutes (mean $\pm \mathrm{SD}$ ) from three experiments. (c) FITC-labeled OspA from B. burgdorferi N40 was used to probe TGE-coated, tick salivary gland extract-coated (SG), BSA-coated, gelatin-coated (G), and FCS-coated (FCS) wells. In some studies, TGE was first treated with a protease inhibitor cocktail (TGE-PI) before incubation with OspA. Bars represent the $\mathrm{OD}_{450}$ at 15 minutes (mean $\pm \mathrm{SD}$ ) from three experiments. 

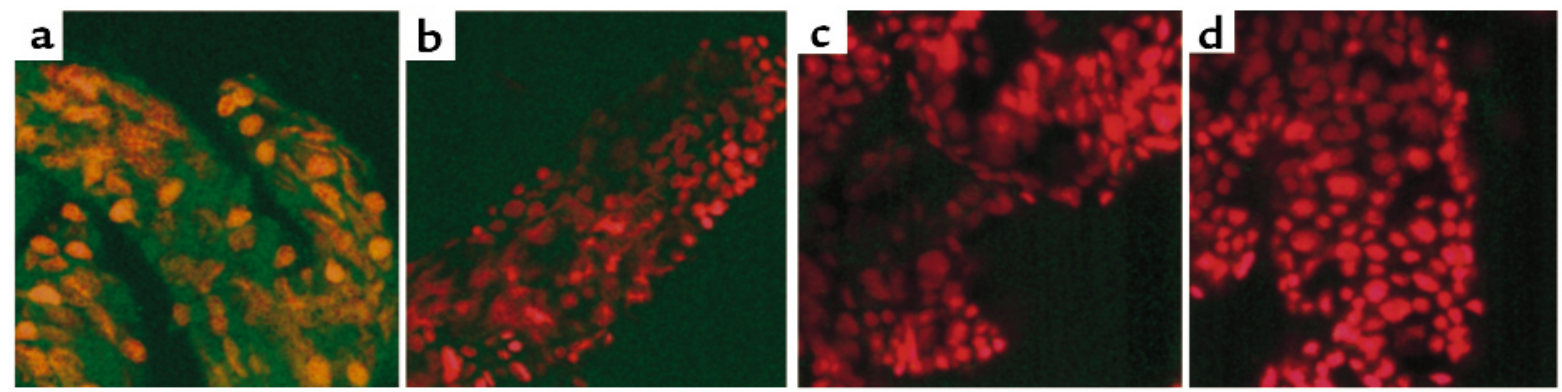

Figure 2

OspA directly binds to the I. scapularis gut. Direct binding of FITC-labeled OspA (a) to the intact unfixed tick gut was detected using confocal microscopy. FITC-labeled OspC (b), FITC-labeled BSA (c), and FITC (d) were used as controls. After probing the tick gut with FITClabeled protein (shown in green), the tissues were stained with propidium iodide to localize the nuclei of the gut cells (shown in red). The FITC and propidium iodide images were examined at $\times 630$ and presented as a single image for clarity.

allowed to air dry. Each slide was then washed three times with PBS and probed with FITC-labeled OspA, OspC, or BSA $(50 \mu \mathrm{L}$ of $100 \mu \mathrm{g} / \mathrm{mL}$ of FITC-labeled protein). Using a conventional Zeiss Axioskop fluorescence microscope, spirochetes in ten microscope fields (100× objective) were examined.

Tick gut: fractionation, and treatment with lipase, glycosidase, or trypsin

Centricon concentrator tubes (Amicon Inc., Beverly, Massachusetts, USA) with different molecular mass cutoffs $(10,30,50$, and $100 \mathrm{kDa})$ were used to separate TGE into different-sized fractions. Equal amounts of protein $(0.5 \mu \mathrm{g})$ from the filtrate and retentate were used to coat microtiter wells that were then probed with labeled OspA as described for ELISA.

TGE was prepared as described, and equal aliquots were incubated with either heat-inactivated $\left(95^{\circ} \mathrm{C}\right.$ for 10 minutes) or active lipase or glycosidases as follows: wheatgerm lipase at $10 \mathrm{U} / \mathrm{mL}$ (Sigma Chemical Co.) for 1 hour at $37^{\circ} \mathrm{C} ; N$-glycosidase F, endo- $\alpha-N$-acetygalactosaminidase, $\alpha-2-3,6,8$-neuraminidase, $\beta-1,4$ galactosidase, and $\beta-N$-acetylglucosaminidase treatment according to a glycoprotein deglycosylation kit (Calbiochem-Novabiochem Corp., San Diego, California, USA). An equal aliquot of TGE was also incubated with trypsin for 1 hour at $37^{\circ} \mathrm{C}$ at $10 \mu \mathrm{g} / \mathrm{mL}$ (Sigma Chemical Co.) in the presence or absence of $20 \mu \mathrm{g} / \mathrm{mL}$ soybean trypsin inhibitor (Sigma Chemical Co.). After enzyme incubation, the TGE with inactivated enzyme was coated onto microtiter plates and probed with labeled OspA as described for ELISA. In some studies, $0.5 \mu \mathrm{g}$ of TGE was first used to coat the microtiter plate wells, and then the plates were washed with PBS and the nonspecific sites were blocked by incubating with $15 \%$ FBS for 2 hours at $37^{\circ} \mathrm{C}$. Then the wells were incubated with trypsin for 1 hour at $37^{\circ} \mathrm{C}$ (with and without presence of soybean trypsin inhibitor). Plates were then probed with labeled OspA. a

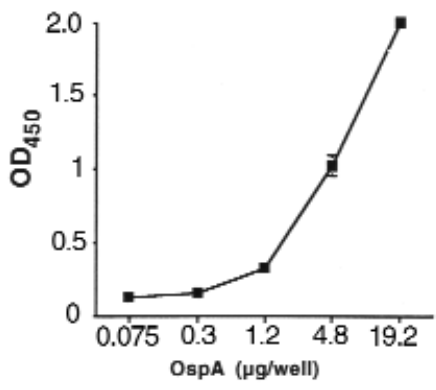

b

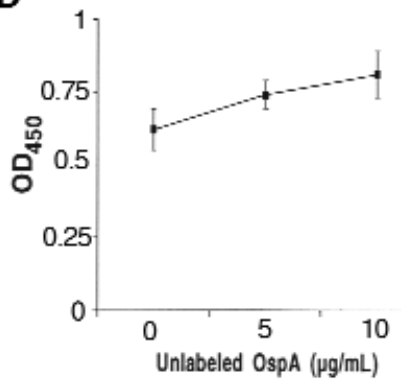

C

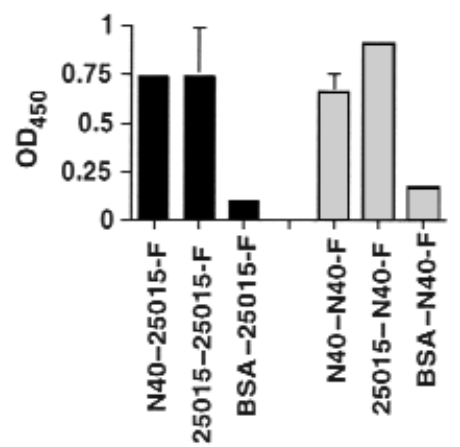

\section{Figure 3}

Effect of increasing OspA on OspA-TGE binding, and OspA-OspA binding. (a) OspA binding to TGE is not saturable. OD was measured at 5 minutes. (b) Effect of excess unlabeled OspA on the binding of labeled OspA to TGE. TGE was probed with FITC-labeled OspA and increasing amounts of unlabeled OspA. These data represent the mean \pm SD from three experiments. OD values were measured at 15 minutes. (c) Binding of OspA with OspA. OspA from B. burgdorferi N40, B. burgdorferi 25015, or BSA was probed with FITC-labeled OspA (25015-F, black bars; or N40-F, gray bars). Bars represent the mean \pm SD from three experiments. OD values were measured at 15 minutes. 


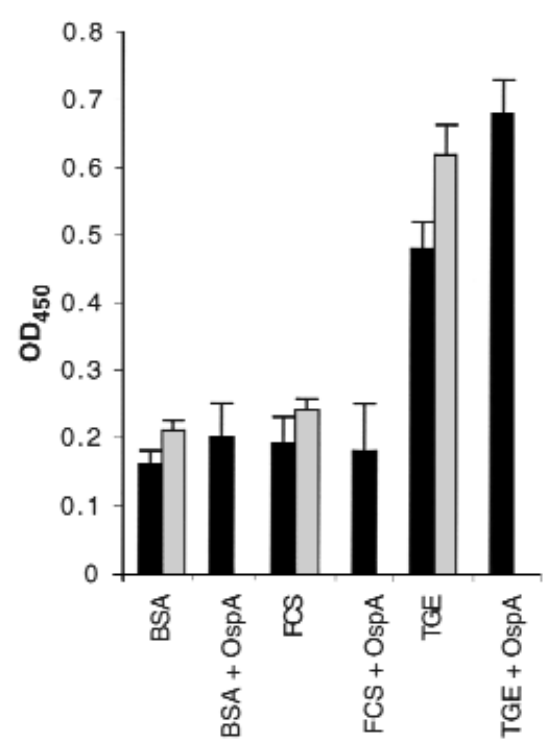

Figure 4

B. burgdorferi $\mathrm{N} 40$ binding to tick gut extract is enhanced in the presence of OspA. BSA-, FCS-, and TGE-coated wells were incubated with $10^{7}$ spirochetes per well in the absence or presence of OspA (example, TGE + OspA). Spirochetes suspended in PBS-Tween were probed with a FlaB mAb (black bars). Spirochetes suspended in PBS were probed with $B$. burgdorferi antisera (gray bars). Bars represent the mean \pm SD from three experiments.

\section{Results}

OspA adherence to a TGE was examined by ELISA. Binding of recombinant FITC-labeled OspA from B. burgdorferi N40, a prototypic B. burgdorferi sensu stricto isolate, and B. burgdorferi 25015, an isolate that has an OspA with variability in the epitopes involved in protective immunity (27), was assessed. Both OspAs readily bound to the TGE (Figure 1a). In addition, OspA bound to TGE in a dose-dependent manner (Figure $1 \mathrm{~b}$ ). Other proteins, such as BSA or B. burgdorferi OspC adhered poorly to the TGE (Figure 1, a and b). The binding appeared to be specific because OspA bound only weakly to a tick salivary gland extract and did not bind to BSA, gelatin, or antigens in FCS (Figure 1c). Furthermore, initial treatment of the TGE with a combination of proteases did not influence OspA-TGE adherence (Figure 1c). OspA also adhered directly to intact unfixed tick gut tissue, as shown by confocal microscopy (Figure 2). In contrast FITC-labeled OspC, FITC-labeled BSA, and FITC did not bind to the tick gut (Figure 2).

The influence of increasing concentrations of OspA on OspA attachment to TGE was then evaluated. The binding of FITC-labeled OspA to the TGE was augmented when increasing amounts of labeled protein were added and could not be saturated (Figure $3 a$ ). In Figure $3 \mathrm{a}$, the OD was recorded at 5 minutes because the value for $19.2 \mu \mathrm{g} /$ well OspA reached the upper limits of detection. At this interval, the OD for 1.2 $\mu \mathrm{g} /$ well OspA was only 0.3 ; however, this value increases to 0.74 when the plates are incubated for 15 minutes, consistent with the data in Figure 1. A sim- ilar pattern, with lower OD values, was apparent when the amount of immobilized TGE was reduced from $0.5 \mu \mathrm{g} /$ well to $0.5 \mathrm{ng} /$ well, and binding was no longer detectable when $0.5 \mathrm{pg} /$ well of TGE was used to coat the plates (data not shown). The inability to saturate OspA binding could be due to a ubiquitous tick gut receptor or to OspA-OspA interactions. The binding of labeled OspA to the TGE did not decrease in the presence of excess unlabeled OspA, suggesting that OspA bound to OspA (Figure 3b). OspA-OspA interactions were therefore directly examined. Labeled OspAs from B. burgdorferi 25015 or N40 specifically bound to unlabeled OspAs (Figure 3c). Studies then assessed this phenomenon using whole B. burgdorferi and the TGE. As expected, spirochetes adhered to the TGE-coated, but not to BSA- or FCScoated, wells (Figure 4). Spirochete suspended in PBS could be detected with the $B$. burgdorferi antisera, whereas bacteria suspended in PBS-Tween were also identified with the FlaB mAb. The addition of OspA enhanced B. burgdorferi binding to the TGE, consistent with the data on OspA attachment to the TGE (Figure 4). In contrast, the addition of OspA did not result in adherence of $B$. burgdorferi to BSA or FCS (Figure 4). OspA binding to B. burgdorferi N40 was then directly demonstrated by immunofluorescence (Figure 5). OspA did not effectively bind to B. burgdorferi 46047 that lack OspA, further suggesting that the
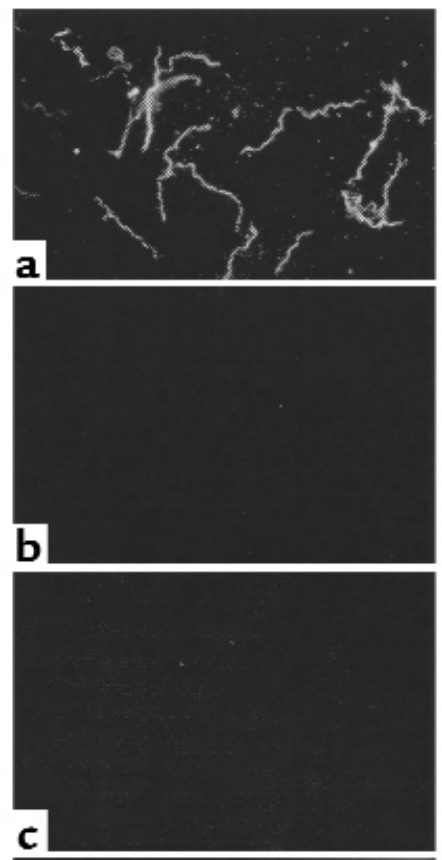

Figure 5

OspA directly binds to $B$. burgdorferi. FITC-labeled OspA (a) was used to directly probe $B$. burgdorferi N40. FITC-labeled OspC (b) and FITC-labeled BSA (c) were used as controls to probe B. burgdorferi N40. FITClabeled OspA was also used to probe $B$. burgdorferi 46047 , an isolate that lacks OspA (d). 


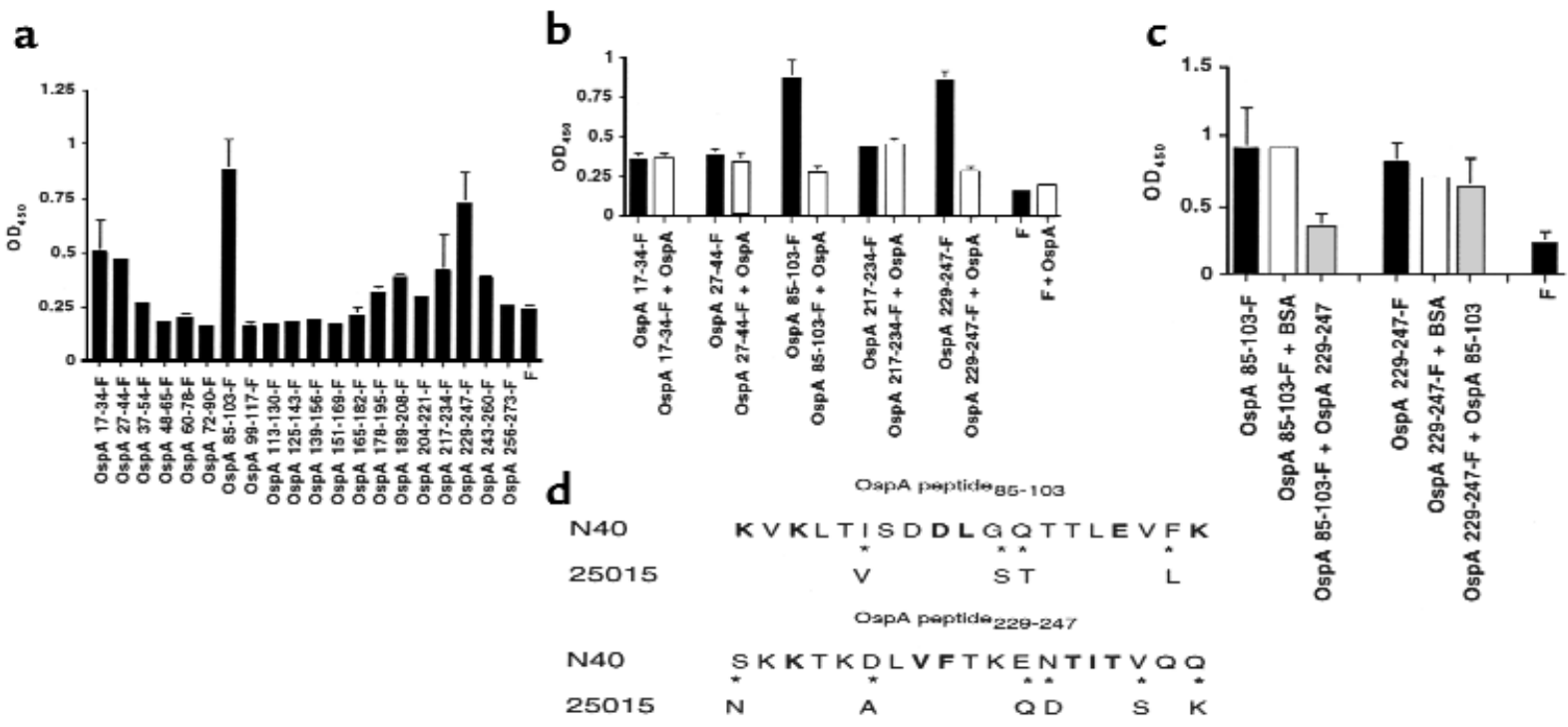

Figure 6

OspA epitopes involved in binding. (a) Relative binding of overlapping OspA peptides ( $100 \mu \mathrm{L}$ of $5 \mu \mathrm{g} / \mathrm{mL}$ peptide) to TGE. The background binding of FITC (F) to TGE has been indicated. The mean \pm SD of three experiments is shown. (b) Inhibition of labeled OspA peptide binding to TGE by unlabeled OspA. TGE was probed with $100 \mu \mathrm{L}$ of FITC-labeled OspA peptide $(5 \mu \mathrm{g} / \mathrm{mL})$ in the absence (black bars) or presence (white bars) of $250 \mu \mathrm{g} / \mathrm{mL}$ of unlabeled OspA. The mean $\pm \mathrm{SD}$ of two experiments using OspA from B. burgdorferi N40 is shown. (c) $\mathrm{OspA}_{229-247}$ and peptide OspA $\mathrm{As}_{8-103}$ bind to the same site, or effectively inhibit binding of a second site, in the tick gut. TGE was probed with $20 \mu \mathrm{g} / \mathrm{mL}$ of FITC-labeled OspA ${ }_{85-103}$ or OspA $229-247$ in the absence (black bars) or presence of competitor, $1 \mathrm{mg} / \mathrm{mL}$ of unlabeled OspA $229-247$ or OspA $A_{85-103}$, respectively (gray bars). Control studies used BSA as a competitor (white bars). The mean \pm SD of six experiments is shown. (d) Amino acid polymorphism in $\mathrm{OspA}_{85-103}$ and $\mathrm{OspA}_{229-247}$. Comparison of the B. burgdorferi $\mathrm{N} 40$ and B. burgdorferi 25015 sequences (variable amino acids are indicated by asterisks). Seventy-nine OspA sequences available in GenBank were aligned, and the strictly conserved residues are presented in bold face. Single-letter abbreviations for the amino acid residues are indicated in Table 1.

binding was specific for OspA (Figure 5). These data demonstrated that B. burgdorferi OspA bound to itself as well as to the tick gut.

The OspA epitope or epitopes that bind TGE were then mapped using 20 overlapping peptides (Table 1 ). The first 16 amino acids of OspA, which encode a signal sequence, were not included in the studies. OspA $\mathrm{A}_{85-103}$ and $\mathrm{OspA}_{229-247}$ strongly bound to the TGE, and several other peptides $\left(\mathrm{OspA}_{17-34}, \mathrm{OspA}_{27-44}\right.$, and OspA $\mathrm{A}_{217-234}$ ) demonstrated moderate binding (Figure $6 a)$. These peptides did not bind to BSA- or serumcoated wells (data not shown). OspA from B. burgdorferi $\mathrm{N} 40$ competed for binding with $\mathrm{OspA}_{85-103}$ and $\mathrm{OspA}_{229-247}$ but not with the other three peptides, suggesting that $\mathrm{OspA}_{85-103}$ and $\mathrm{OspA}_{229-247}$ contained functional binding sites (Figure $6 \mathrm{~b}$ ). Similar results

\section{Figure 7}

Binding of OspAs with mutations within amino acids $85-103$ or 229-247 to TGE. Site-directed mutagenesis was performed to generate OspAs, designated M1-M6 with substitution in amino acids that are conserved between B. burgdorferi species within region 85-103 and 229-247 (Figure 6d). M1-M6 contain the following amino acid replacements: M1 (KVK85-87 to $\triangle E V E$ ), M2 (DL93-94 to $\triangle \mathrm{AR}$ ), M3 (EVFK100-103 to $\triangle$ AVFA), M4 (K231 to $\triangle \mathrm{A}$ ), M5 (VF236-237 to $\triangle \mathrm{GS}$ ), and M6 (TIT242-244 to $\triangle \mathrm{ANA}$ ). Binding of OspA and M1-M6 to TGE is depicted. $B$ represents binding of OspA to BSA (control). M1-M6 showed a similar degree of binding to BSA (data not shown). Bars represent the mean \pm SD from four experiments. ${ }^{A} P<0.001$. were obtained when OspA from B. burgdorferi 25015 was used for competition (data not shown). To test whether OspA $\mathrm{A}_{85-103}$ and $\mathrm{OspA}_{229-247}$ adhered to the same TGE site, competition studies were performed using FITC-labeled $\mathrm{OspA}_{85-103}$ or $\mathrm{OspA}_{229-247}$ and excess unlabeled OspA ${ }_{85-103}$ or $\mathrm{OspA}_{229-247}$. OspA $229-247$ strongly competed for binding with $\mathrm{OspA}_{85-103}$ but not vice versa (Figure 6c). This suggests either that (a) the

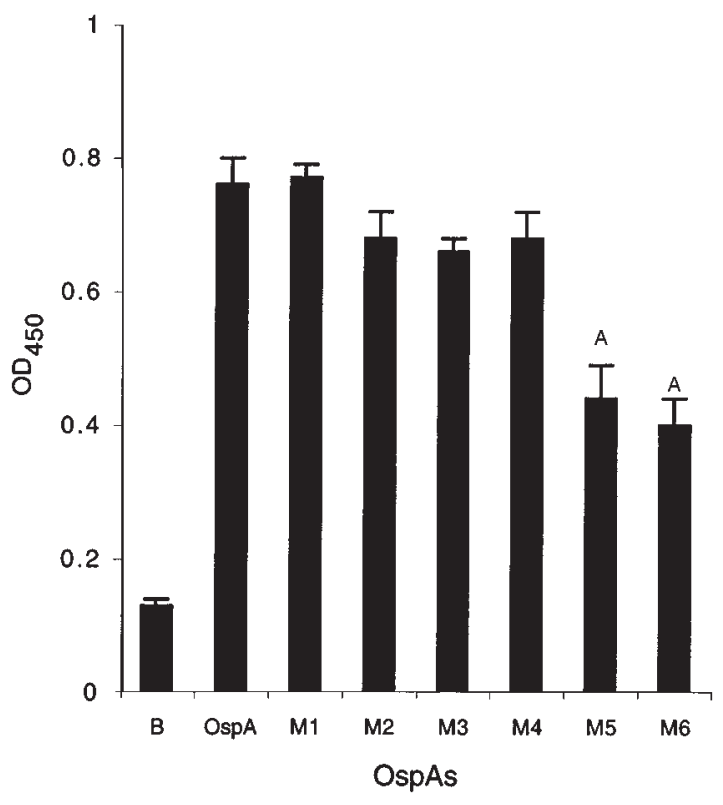


two peptides bind to the same site in the tick gut but that OspA $229-247$ binds with a much higher affinity than OspA $\mathrm{A}_{8-103}$ or that (b) OspA $229-247$ can effectively block the binding at a second site.

OspAs from B. burgdorferi N40 and 25015 were both able to bind to TGE. The OspA $85-103$ and OspA $229-247$ sequences of these spirochetes were therefore compared. Conserved amino acid positions within these regions of B. burgdorferi $\mathrm{N} 40$ and 25015 may possibly facilitate OspA adherence to the tick gut (Figure 6d). Site-directed mutagenesis was accordingly used to generate OspAs with amino acid substitutions in these regions, for use in TGE binding assays. Six mutant OspAs, three with substitutions in $\mathrm{OspA}_{85-103}$ and three with alterations in OspA 229-247, were created that encompass all the conserved amino acids between $B$. burgdorferi species (see Methods and Figure 6d). OspA mutants M5 and M6, with replacements in amino acids $236-7$ or $242-4$ of OspA $229-247$, respectively, demonstrated significantly less $(P<0.001)$ binding to TGE than did authentic OspA (Figure 7).

The OspA receptor in the tick was then partially characterized. To determine the approximate molecular mass of the ligand, size-separated fractions of the TGE were used in ELISA. A ligand or ligand complex greater than $100 \mathrm{kDa}$ exhibited binding activity (Figure 8a). To understand the biochemical nature of the OspA binding moiety, the TGE was treated with wheatgerm lipases, a variety of glycosidases ( $N$-glycosidase F, endo- $-N$-acetygalactosaminidase, $\alpha 2$ $3,6,8$-neuraminidase, $\beta 1,4$-galactosidase, and $\beta-N$ acetylglucosaminidase), or trypsin. Heat-denatured enzyme (in the case of lipases or glycosidases) or proteolytic enzyme in the presence of specific inhibitor (soybean trypsin inhibitor) served as controls. Lipase or glycosidases did not affect OspA binding to the TGE (data not shown). In contrast, pretreatment of the TGE with trypsin markedly diminished OspA adherence (Figure 8b). A similar reduction in OspA binding was also observed when the TGE immobilized on microtiter wells was treated with trypsin (Figure 8b, lanes marked W). OspA therefore bound to a high molecular mass protein, or protein receptor complex, in the TGE.

\section{Discussion}

These data demonstrate that B. burgdorferi OspA specifically binds to a protein or proteins in the gut of I. scapularis. Selective ospA expression as the spirochete enters larval ticks may allow B. burgdorferi to adhere to and colonize the vector. Similarly, repression of ospA during tick feeding may facilitate $B$. burgdorferi detachment from the gut and migration to the salivary glands and ultimately the vertebrate host. The weak binding of OspA to tick salivary glands (Figure $1 \mathrm{~b}$ ) suggests that the tick receptor that binds OspA may be present, at least at some level, in this tissue as well. However, spirochetes that migrate from the tick gut to the salivary gland rarely, if ever, express ospA $(7,9,10)$. Therefore, any degree of
OspA-salivary gland binding is not likely to be functionally relevant. These studies also indicate that OspAOspA interactions may increase spirochete adherence in the gut through self-binding, particularly at high $B$. burgdorferi density, and this phenomenon may partially explain the reported in vivo aggregation of $B$. burgdorferi in the tick gut or on cell lines in vitro (29-33). The downregulation of OspA by spirochetes during the bloodmeal may also facilitate dissemination to the tick salivary glands and the host's dermis by preventing the bacteria from clumping to one another during transmission.

The crystal structure of OspA has recently been elucidated and demonstrates that OspA $229-247$ extends from $\beta$-strand 19 to 20 in the $\mathrm{COOH}$-terminal barrel sheet (34). Interestingly, Phe-237 and Ile-243, within OspA $229-247$, are associated with a hydrophobic_cavity suggested to be a ligand-binding site, and protein folding brings residues $236-7$ and $242-4$ into close prox-

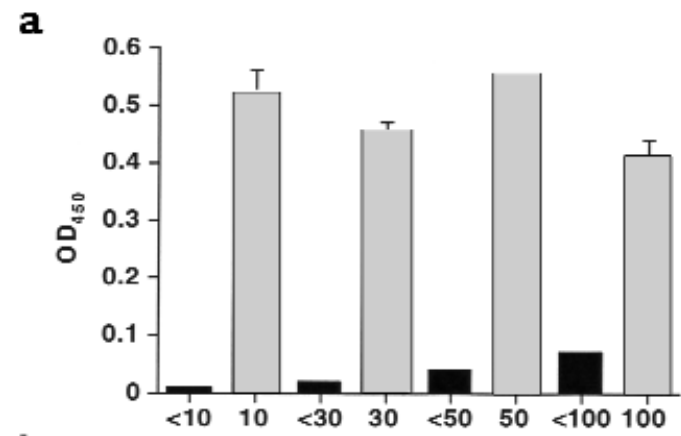

b

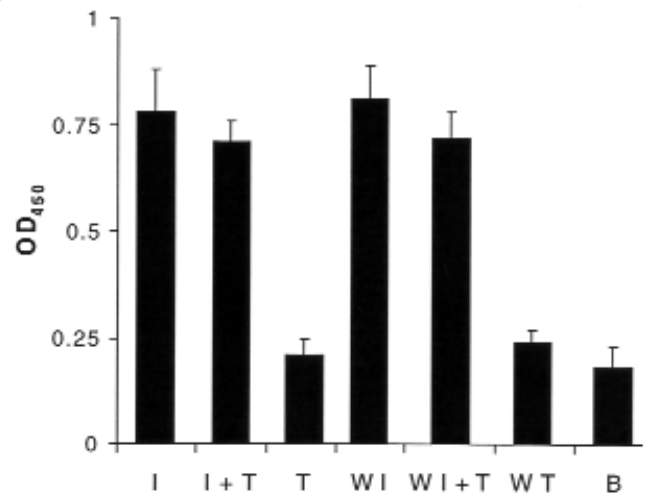

Figure 8

Characterization of the tick gut receptor that binds OspA. (a) Binding of OspA to different-sized TGE fractions. Tick gut extracts were subjected to size fractionation using Centricon concentrator tubes with molecular mass cutoffs of either $10,30,50$, or $100 \mathrm{kDa}$. In each separate fractionation, black and gray bars represent the separated TGE containing the smaller and larger material, respectively. The means \pm SD from two experiments are shown. (b) Effect of trypsin treatment on OspA binding to TGE. TGE was treated with soybean trypsin inhibitor (I), soybean trypsin inhibitor and trypsin $(I+T)$, or trypsin $(T)$. The trypsin-treated and control TGEs were then used to coat microtiter wells and probed with labeled OspA. The OD of the binding of labeled OspA to BSA is also shown as a control (B). In an additional series of experiments, marked "W," TGE-coated microtiter wells were treated with soybean trypsin inhibitor, soybean trypsin inhibitor and trypsin, or trypsin. Bars represent the mean \pm SD from three experiments. 
imity (34). The site-directed mutagenesis studies (Figure 7) further suggest that this region of $\mathrm{OspA}_{229-247}$ is important in OspA-TGE binding because substitutions in amino acids $236-7$ or 242-4 significantly reduced the binding to OspA to TGE. The reduced degree of binding could be due to the replacement of amino acids that are directly involved in attachment, or in the alteration of the structure of the putative hydrophobic cavity that is the probable ligand binding domain. The fact that OspA molecules are capable of adhering to each other must also be taken into consideration in the analysis of the mutagenesis studies. The OspA mutants M5 and M6 may have even greater defects in attachment to the tick gut because OspAOspA binding may mask a further decrease in OspAtick gut adherence. Nevertheless, the significant decrease in binding of M5 and M6, with substitutions in amino acids 236-7 and 242-4, further demonstrates that the attachment of OspA to the tick gut is specific and, at least in part, mediated by the putative binding pocket in the $\mathrm{COOH}$-terminus of the protein.

The role of OspA during mammalian infection is not yet known; however, one report has suggested that OspA may facilitate spirochete interactions with endothelium (35). Given that $\operatorname{csp} A$ is not generally expressed during human and murine infection, it is possible that OspA does not have an important role in the vertebrate host. Alternatively, reports have demonstrated ephemeral OspA antibody and T-cell responses during early-stage Lyme disease, and OspA responses have been identified during chronic antibiotic-resistant Lyme arthritis, suggesting that OspA may sometimes be present during infection (16-21). One speculation from these efforts is that OspA may play a role in persistent $B$. burgdorferi colonization of synovial tissue. Identification of the I. scapularis ligand(s) that binds to OspA in the tick may provide clues to additional ligands in the mammalian host that could also potentially interact with this lipoprotein.

B. burgdorferi has exquisitely adapted to survive in both the arthropod vector and vertebrate host, and we show that OspA is a spirochete antigen that facilitates adherence in I. scapularis. In the mammalian host, several spirochete antigens are involved in attachment to the host. For example, decorin-binding protein (Dbp) A mediates that binding of B. burgdorferi to decorin, and BBK32 promotes the binding of spirochetes to fibronectin (36-38). In I. scapularis, B. burgdorferi resides in specific locations during distinct intervals of infection, including the gut of unfed ticks and the salivary glands of engorging tick, and it is possible that different spirochete antigens assist in the colonization of different tissues within the vector. The present studies with OspA were performed at $37^{\circ} \mathrm{C}$, whereas the ambient temperature in flat ticks is lower $\left(20^{\circ} \mathrm{C}\right)$, and the temperature in engorging ticks and mammals is closer to $37^{\circ} \mathrm{C}$; therefore, it is likely that temperature and other host factors can further influence specific B. burgdorferivector/vertebrate interactions. Understanding the function of $B$. burgdorferi genes that are selectively expressed throughout the spirochete life cycle, and the molecular basis of vector-B. burgdorferi interactions, will expedite new approaches to the control of Lyme disease and provide a model for other arthropod-borne infections.

\section{Acknowledgments}

We thank P. Voet and M. Vasil for assistance and A. van der Ende and A.P. van Dam for reviewing the manuscript.

This work was supported by grants from NIH, the Arthritis Foundation, the American Heart Association, the Eshe and Mathers Foundations, the Community Foundation of Greater New Haven, and a gift from SmithKline Beecham Biologicals. E. Fikrig is the recipient of a Burroughs Wellcome Clinical Scientist Award in Translational Research.

1. Nadelman, R.B., and Wormser, G.P. 1998. Lyme borreliosis. Lancet. 352:557-565.

2. Barbour, A.G., and Fish, D. 1993. The biological and social phenomenon of Lyme disease. Science. 260:1610-1616.

3. Fikrig, E., Barthold, S.W., Kantor, F.S., and Flavell, R.A. 1990. Protection of mice against the Lyme disease agent by immunizing with recombinant OspA. Science. 250:553-556.

4. Schaible, U.E., et al. 1990. Monoclonal antibodies specific for the outer surface protein A (OspA) of Borrelia burgdorferi prevent Lyme borreliosis in severe combined immunodeficiency (scid) mice. Proc. Natl. Acad. Sci. USA. 87:3768-3772.

5. Sigal, L.H., et al. 1998. A vaccine consisting of recombinant Borrelia burgdorferi outer-surface protein A to prevent Lyme disease. N. Engl.J. Med. 339:216-222.

6. Steere, A.C., et al. 1998. Vaccination against Lyme disease with recombinant Borrelia burgdorferi outer-surface lipoprotein A with adjuvant. N. Engl. J. Med. 339:209-215.

7. de Silva, A.M., Telford, S.R., Brunet, L.R., Barthold, S.W., and Fikrig, E. 1996. Borrelia burgdorferi OspA is an arthropod-specific transmission-blocking Lyme disease vaccine. J. Exp. Med. 183:271-275.

8. de Silva, A.M., Fish, D., Burkot, T.R., Zhang, Y., and Fikrig, E. 1997. OspA antibodies inhibit the acquisition of Borrelia burgdorferi by Ixodes ticks. Infect. Immun. 65:3146-3150.

9. Coleman, J.L., et al. 1997. Plasminogen is required for efficient dissemination of Borrelia burgdorferi in ticks and for enhancement of spirochetemia in mice. Cell. 89:1111-1119.

10. Schwan, T.G., Piesman, J., Golde, W.T., Dolan, M.C., and Rosa, P.A. 1995. Induction of an outer surface protein on Borrelia burgdorferi during tick feeding. Proc. Natl. Acad. Sci. USA. 92:2909-2913.

11. Montgomery, R.R., Malawista, S.E., Feen, K.J., and Bockenstedt, L.K. 1996. Direct demonstration of antigenic substitution of Borrelia burgdorferi ex vivo: exploration of the paradox of the early immune response to outer surface proteins $\mathrm{A}$ and $\mathrm{C}$ in Lyme disease. J. Exp. Med. 183:261-269.

12. Gern, L., Schaible, U.E., and Simon, M.M. 1993. Mode of inoculation of the Lyme disease agent Borrelia burgdorferi influences infection and immune responses in inbred strains of mice. J. Infect. Dis. 167:971-975.

13. Schaible, U.E., et al. 1993. Distinct patterns of protective antibodies are generated against Borrelia burgdorferi in mice experimentally inoculated with high and low doses of antigen. Immunol. Lett. 36:219-226.

14. de Silva, A.M., et al. 1998. Immune evasion by tick-borne and hostadapted Borrelia burgdorferi. J. Infect. Dis. 177:395-400.

15. Fikrig, E., et al. 1999. Borrelia burgdorferi erpT expression in the arthropod vector and murine host. Mol. Microbiol. 31:281-290.

16. Kalish, R.A., Leong, J.M., and Steere, A.C. 1995. Early and late antibody responses to full-length and truncated constructs of outer surface protein A of Borrelia burgdorferi in Lyme disease. Infect. Immun. 63:2228-2235.

17. Schutzer, S.E., et al. 1997. Simultaneous expression of Borrelia OspA and $\mathrm{OspC}$ and IgM response in cerebrospinal fluid in early neurologic Lyme disease. J. Clin. Invest. 100:763-767.

18. Schutzer, S.E., Coyle, P.K., Dunn, J.J., Luft, B.J., and Brunner, M. 1994. Early and specific antibody responses to OspA in Lyme disease. J. Clin. Invest. 94:454-457.

19. Akin, E., McHugh, G.L., Flavell, R.A., Fikrig, E., and Steere, A.C. 1999. The immunoglobulin (IgG) antibody response to OspA and OspB correlates with severe and prolonged Lyme arthritis and the IgG response to P35 correlates with mild and brief arthritis. Infect. Immun. 
67:173-181.

20. Kalish, R., Leong, J.M., and Steere, A.C. 1993. Association of treatment-resistant chronic Lyme arthritis with antibody reactivity to OspA and OspB of Borrelia burgdorferi. Infect. Immun. 61:2774-2779.

21. Gross, D.M., et al. 1998. Identification of LFA-1 as a candidate autoantigen in treatment-resistant Lyme arthritis. Science. 281:703-706.

22. Barthold, S.W., Fikrig, E., Bockenstedt, L.K., and Persing, D.H. 1995. Circumvention of outer surface protein A immunity by host-adapted Borrelia burgdorferi. Infect. Immun. 63:2255-2261.

23. Barbour, A.G. 1984. Isolation and cultivation of Lyme disease spirochetes. Yale J. Biol. Med. 57:521-525.

24. Anderson, J.F., et al. 1996. Novel Borrelia burgdorferi isolates from Ixodes scapularis and Ixodes dentatus ticks feeding on humans. J. Clin. Microbiol. 34:524-529.

25. Bockenstedt, L.K., Fikrig, E., Barthold, S.W., Flavell, R.A., and Kantor, F.S. 1996. Identification of a Borrelia burgdorferi OspA T cell epitope that promotes anti-OspA IgG in mice. J. Immunol. 157:5496-5502.

26. Bockenstedt, L.K., et al. 1997. Borrelia burgdorferi strain-specific Osp C-mediated immunity in mice. Infect. Immun. 65:4661-4667.

27. Fikrig, E., et al. 1992. Borrelia burgdorferi strain 25015: characterization of outer surface protein $\mathrm{A}$ and vaccination against infection. $J$. Immunol. 148:2256-2260.

28. Sadziene, A., Thomas, D.D., Bundoc, V.G., Holt, S.C., and Barbour, A.G. 1991. A flagella-less mutant of Borrelia burgdorferi. Structural, molecular, and in vitro functional characterization. J. Clin. Invest. 88:82-92.

29. Burgdorfer, W., et al. 1982. Lyme disease: a tickborne spirochetosis? Science. 216:1317-1319.
30. Kurtti, T.J., Munderloh, U.G., Krueger, D.E., Johnson, R.C., and Schwan, T.G. 1993. Adhesion to and invasion of cultured tick (Acarina: Ixodidae) cells by Borrelia burgdorferi (Spirochaetales: Spirochaetaceae) and maintenance of infectivity. J. Med. Entomol. 30:586-596.

31. Thomas, D.D., and Comstock, L.E. 1989. Interaction of Lyme disease spirochetes with cultured eukaryotic cells. Infect. Immun. 57:1324-1326.

32. Garcia-Monco, J.C., Fernandez-Villar, B., and Benach, J.L. 1989. Adherence of the Lyme disease spirochete to glial cells and cells of glial origin. J. Infect. Dis. 160:497-506.

33. Garcia-Monco, J.C., and Benach, J.L. 1995. Lyme neuroborreliosis. Ann. Neurol. 37:691-702.

34. Li, H., Dunn, J.J., Luft, B.J., and Lawson, C.L. 1997. Crystal structure of Lyme disease antigen outer surface protein A complexed with an Fab. Proc. Natl. Acad. Sci. USA. 94:3584-3589.

35. Comstock, L.E., Fikrig, E., Shoberg, R.J., Flavell, R.A., and Thomas, D.D. 1993. A monoclonal antibody to OspA inhibits association of Borrelia burgdorferi with human endothelial cells. Infect. Immun. 61:423-431.

36. Guo, B.P., Brown, E.L., Dorward, D.W., Rosenberg, L.C., and Hook, M. 1998. Decorin-binding adhesins from Borrelia burgdorferi. Mol. Microbiol. 30:711-723.

37. Guo, B., Norris, S.J., Rosenberg, L.C., and Hook, M. 1995. Adherence of Borrelia burgdorferi to the proteoglycan decorin. Infect. Immun. 63:3467-3472.

38. Probert, W.S., and Johnson, B.J. 1998. Identification of a $47 \mathrm{kDa}$ fibronectin-binding protein expressed by Borrelia burgdorferi isolate B31. Mol. Microbiol. 30:1003-1015 